\title{
The Use of Fluorescein in Lepra Reaction.
}

Gordon A. RYRIE.

DHE Federal Leper Settlement, Sungei Buloh, F.M.S., treats over one hundred and fifty cases of severe lepra 1 reaction a year. These cases are of varied nationality and they are studied and treated under modern hospital conditions; they are institution patients whose previous condition is already known and recorded. For over a year the majority of such cases have been treated by the intravenous injection of fluroescein and we are now able to make a survey of the effects of this treatment.

A number of difficulties arise in assessing the effect of any drug in reaction. Lepra reaction is observed here to be very different in its racial severity. It is seen for instance in a much more virulent form among Chinese than among Indians who are living in the settlement under the same conditions. Further the syndrome known as reaction almost certainly includes a number of different entities which may require varied forms of treatment. The fever is remittent in many cases and remission may coincide with the exhibition of treatment. Reaction may go on for days or for months and may terminate by lysis or crisis. 
THE LEPROSY HOSPIIAL, SHAIKH OTHMAN, AIIEN.

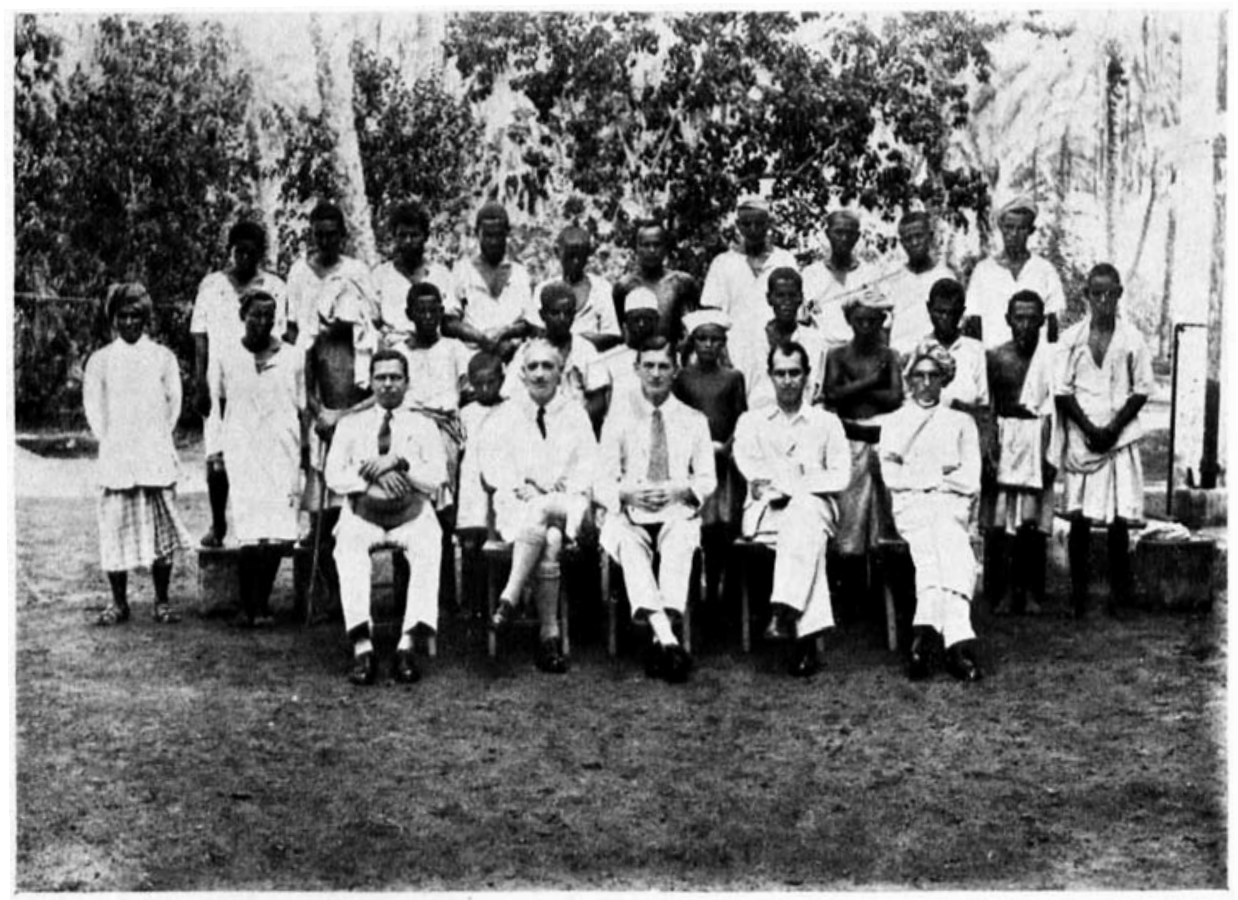

Group of Patients with the Members of the Leprosy Board.

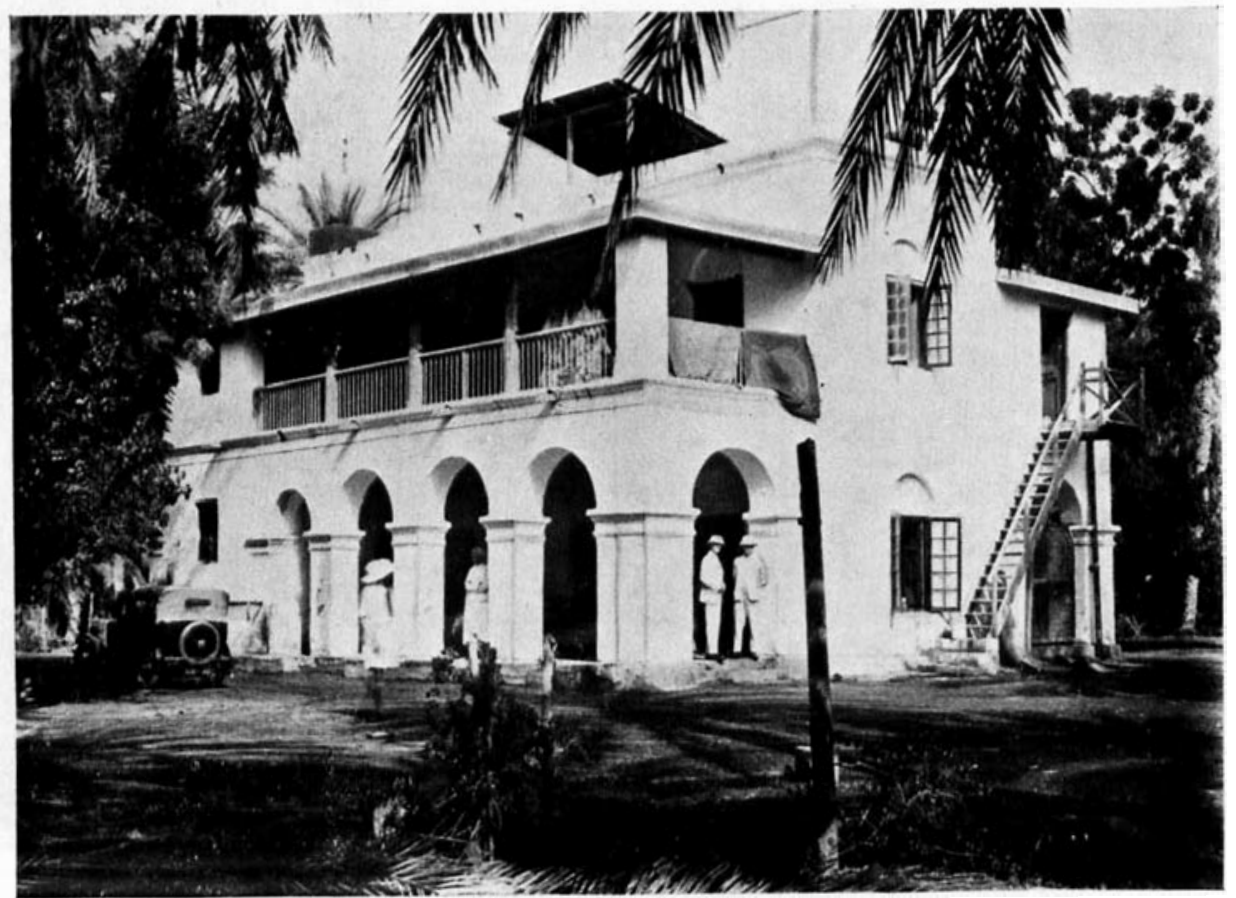

General View of the Hospital Building. 


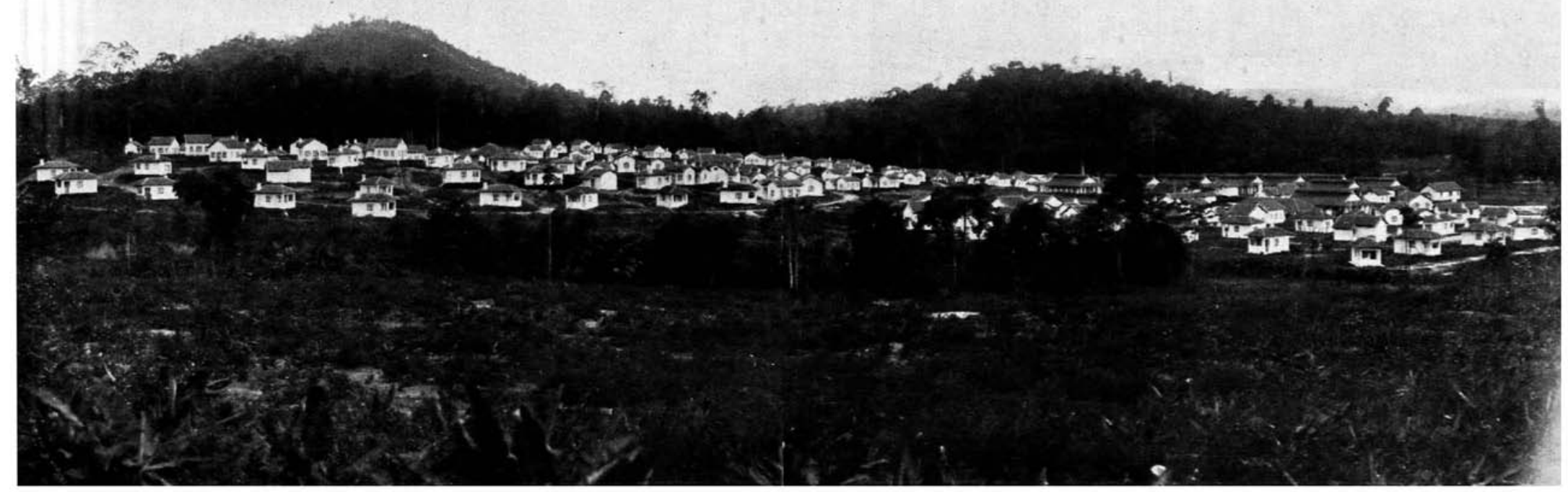

Panoramic View of Leprosy Asylum, Sungei Buloh, Federated Malay Sta:es. 
It is difficult again to estimate the effect of hospital regime. Has your concomitant drug cured the patient or the dose of salts the nurse gave him on admission?

And, lastly, the multitude of preparations that appear to have been used successfully in reaction shows that the psychological effect of new treatment may be considerable.

Fluorescein, along with eosin and rhodamines, is a zanthene dye. Its trial arose from the recent work by Dr. Muir, on mercurochrome summarised in Leprosy in India. January, 1933. Administration of mercurochrome, as pointed out by Muir, often causes a dramatic abortion of the reaction process, and this is attributed to its effect in clearing up septic conditions. This explanation is open to a good deal of theoretical doubt, and I considered that the effect of mercurochrome on reaction was much more likely to be due to its fluorescein content. Such a dye, when injected into the blood stream, is taken up-among the other elements of the reticulo-endothelial system-by the macrophage cells of the leprotic lesion and may then interfere with the process deriving from the attachment of antibody to the cells.

After trial of fluorescein in a number of cases the results were found to be similar to those obtained by the administration of mercurochrome. Fluorescein, however, was found to possess a number of advantages. It can be given safely in much larger doses than mercurochrome, and if administered with proper precautions does not cause a rigor or febrile response.

Fluorescein is obtainable in two forms. Ordinary fluorescein is soluble in alkali, so that the dye should be dissolved in the same percentage strength of bicarbonate of soda. That is, a 2 per cent. solution of fluorescein should be made up by adding the dye to a 2 per cent. solution of bicarbonate of soda in distilled water. Fuorescein-soluble, however, readily dissolves in water and does not require alkali. In most cases we have given 10 c.c. of a 2 per cent. solution intravenously. To obtain the maximum effect the injections should be given twice weekly. The solution should be freshly made with distilled water or saline; it should be boiled and filtered before use. The dye itself should be stored away from the light. The quantity that can be injected varies considerably with different samples of the dye, but I have given 50 c.c. of a 2 per cent. solution without any apparent ill-effects. I have elsewhere considered its effect on ordinary leprotic lesions. 
Out of about eighty cases of severe leprotic reaction treated during the latter part of 1932, thirty-one were given fluorescein. Thirtcen of these, or nearly 42 per cent. showed a rapid recovery after the administration of either one or two injections of 10 c.c. 2 per cent. fluorescein.

Four cases showed no improvement. Fifteen cases, or 45 per cent. showed a decline of fever by lysis after three to six injections.

These results must be subjected to a number of qualifications. With regard to the 42 per cent. who showed rapid defervescence of symptoms-mostly within 48 hours of injection, it must be stated that one or two striking recoveries when this treatment was started made a profound psychological effect on the Chinese patients. A certain amount of subsequent effects must be attributcd to this. Some of this result, in any case, must be " written off " and ascribed to hospital regime and psychotherapy.

The 45 per cent. who showed decline of fever by lysis are even more difficult to assess. It is probable that a number of them would have got better within the same period without fluorescein.

Even when every caution is observed, however, in interpreting results, one is left with considerable evidence that fluorescein exerts a definite and occasionally striking beneficial effect on lepra fever. It was found difficult to persuade control cases to continue with P.A.T. Control cases on mercurochrome appeared as a rule to be quite satisfiedtheir general attitude and the course of their illness being on the whole much the same as those treated with fluorescein. Illustrative cases :

Low Kee, male, Chinese, Aet. 24, C.2 N.1. Admitted $11 / 2 / 33$ with history of reaction for the last four days, the first symptom being pain in the left ulnar nerve. On the third day of illness reaction spots appeared on the right elbow and spread to the face, arms and thighs. Joint pains appeared on the fourth day.

Laboratory report, Wassermann negative, Kahn negative. Blood negative X3 for malaria, stools negative X2. Urine normal, hæmoglobin 65 per cent. serum calcium 9.6.

Precipitating factor-said to be due to taking Tai Foong Chee. Examination of the circulating blood twice weekly, though the illness failed to reveal lepra bacilli, but they were found on the fresh patches in large numbers. Sedimentation rate examined in the ward twice weekly, began at 61 and gradually subsided as the patient improved. 
Temperature.

\section{Conment.-}

A case of decline of reaction after one injection of fluorescein.

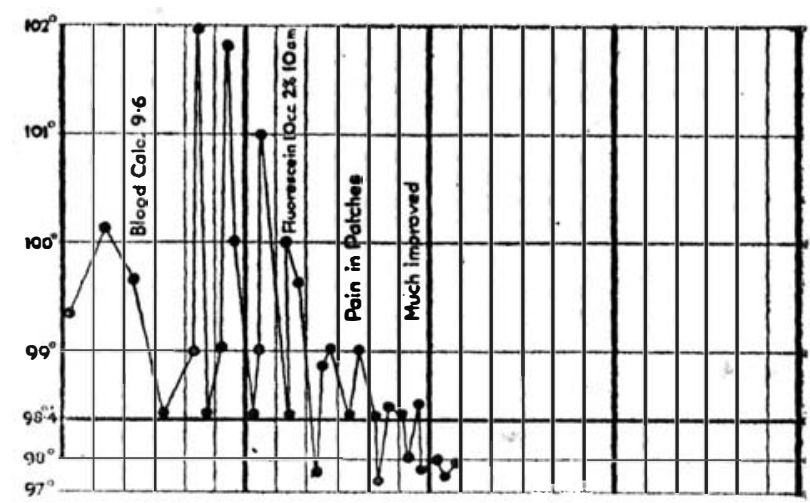

2. Case of Pang Thye, Chinese, female, Aet. 16. Admitted 1/9/32, complaining of fever and reaction of a week's duration. Unable to state the cause and sequence of her symptoms.

Laboratory report: Wassermann + Kahn + Stools A.D.O. Blood' negative $\mathrm{x} 3$ for malaria. Urine specific gravity 1020, with no abnormal constituents. Hæmoglobin 50 per cent.

Examination: Tongue dirty, fauces inflamed, extensive pyorrhœa, right heart enlarged, one-finger spleen, descending colon, palpable mass of fæces.

Temperature swung daily from subnormal in the morning to a mid-day peak between $100 \mathrm{deg}$. and $101 \mathrm{deg}$. Multiple injections of fluorescein made little or no effect on the reaction and the patient only recovered very gradually.

I have quoted this girl's case as typical of a number of cases who are unable to stand the strain that the demands of ordinary outdoor activity make on their poisoned metabolism. The patient naturally had a history of frequent reactions and neither fluorescein nor any other drug used to control lepra fever can deal adequately with the multiple precipitating factors revealed on examination. The subsequent treatment of the precipitating factors in this case (with the exception of attention to bowel hygiene) each caused a fresh exacerbation of the reaction condition.

In a small number of instances a patient who failed to respond to fluorescein has been found to improve rapidly on mercurochrome. In the same way a number who appeared resistant to mercurochrome occasionally benefited by a later injection of fluorescein.

\section{Summary :-}

(1) In a fairly large proportion of cases of lepra fever the administration of fluorescein appears to give benefit. 
(2) Its action, as observed by us, seems similar to that of mercurochrome, which is a fluorescein compound.

(3) It is much less toxic than mercurochrome, can be given in much larger or more frequent doses if desired, and if properly administered causes no febrile reaction.

(4) It may be given in cases of reaction in doses of 10 c.c. of a 2 per cent. solution twice weekly, but we have given larger and more frequent doses without ill effects. It should be freshly prepared.

(5) It is more likely to be effective after a few days of hospital regime, calcium therapy, and a milk and fruit diet. By waiting a few days before administration it is also possible to differentiate to some extent between purely nursing effects and those of the dye.

(6) Moderate disease of the kidneys does not appear to be a contra indication.

(7) Prolonged administration does not appear to harm the patient. 